\title{
REGARDS Sécurité sanitaire des aliments et consommateurs
}

\section{Viewpoint Consumers and food safety}

Oléagineux, Corps Gras, Lipides. Volume 7, Numéro 5, 443-8, Septembre - Octobre 2000, Dossier : Sécurité sanitaire des aliments et industrie

Auteur(s) : Claude FISCHLER, CETSAH, 22, rue d'Athènes, 75009 Paris.

Résumé : Les crises récentes dans le domaine de la sécurité alimentaire, en particulier celle de l'ESB, entraînent dans le monde développé et au-delà de profonds changements, temporaires et/ou permanents, dans les consommations alimentaires et les attitudes qui s'y associent. Entre le public profane et les experts, scientifiques et techniciens, l'appréciation du risque est souvent divergente : les techniciens connaissent le nombre de cas cliniques, la morbidité et la mortalité éventuelle, et s'appuient sur ces données ; le public, lui, ne procède pas nécessairement à une estimation probabiliste du risque. Il est sensible aux images et aux récits médiatiques, à certains types de risques plus qu'à d'autres et il éprouve des difficultés à évaluer le risque " faible ". II est particulièrement sensible aux risques liés à l'alimentation et cette sensibilité traduit certains aspects anxiogènes des filières agro-alimentaires modernes. Ce ne sont pas toujours les dangers quantitativement les plus meurtriers qui provoquent les inquiétudes les plus profondes, les répercussions médiatiques et économiques les plus massives. La perception du risque par le consommateur est en partie prédictible. Les dimensions pertinentes comprennent certaines configurations de risque qui tendent à augmenter l'inquiétude et l'indignation. D'autres facteurs sont liés à des traits psychologiques et cognitifs, dont ce que les anthropologues appellent la " pensée magique ", ainsi qu'à des aspects sociaux et culturels. Toutes les sociétés, tous les groupes sociaux ne craignent pas les mêmes risques.

Mots-clés : risque, perception du risque, habitudes alimentaires, consommation alimentaire, peurs alimentaires, comportement alimentaire. 
Summary : Recent crises associated with food safety issues in the affluent world, particularly the BSE crisis, bring about profound changes, temporary and/or permanent, in food consumption patterns and attitudes. Experts evaluate risks using various probabilistic tools, for example epidemiological data (mortality and morbidity rates associated with a given occurrence). Lay people, however, rather than evaluating risk in a probabilistic fashion, perceive it in ways that are often described as "irrational" by experts, industry and authorities and are sensitive to media coverage. To say the least, experts and consumers often disagree. This is particularly true when it comes to possible dangers associated with food. Food is the one most intimate form of consumption: What we eat enters our body, becomes part of ourself. Thus humans tend to protect their body and their self against possible toxic, dangerous intrusions through food. Perception of risk by the consumer can be in part predicted. Relevant dimensions include risk configuration (certain types and configurations tend to increase public outrage). Other factors are associated with psychological and cognitive features, what anthropologists call "magical thinking", but also social and cultural factors. Not all groups and cultures are equally fearful or worried, and not about the same risks.

Keywords : risk perception, food habits, food consumption.

\section{ARTICLE}

Je commencerai par indiquer que ma recherche porte sur la perception par le public des problèmes des filières alimentaires. Dans cette perspective, il s'agit moins d'évaluer le risque que d'analyser sa perception par le public, perception dont je voudrais vous présenter brièvement les dimensions pertinentes.

Il y a quelques années, un chercheur américain a soumis une liste de dangers et de risques à des échantillons différents : d'un côté, des membres du grand public, en l'occurrence, la League of Women Voters (association d'électrices américaines), un groupe d'étudiants, des membres d'un club sportif et, de l'autre côté, des experts en évaluation des risques, autrement dit des gens du métier, du domaine des assurances ou de la recherche scientifique. II leur a soumis une liste de risques auxquels il leur a demandé d'affecter une note de gravité.

D'importantes divergences se sont exprimées entre les membres de l'association féminine et les experts sur la question de l'énergie nucléaire. Pour le grand public, le premier risque était le risque nucléaire, alors que celui-ci ne venait qu'en vingtième position pour les experts. L'usage abusif des rayons $X$ en médecine apparaissait comme un risque sérieux pour les experts, qui le classaient au septième rang, alors qu'il était à peine mentionné par le grand public qui le mettait en vingt-deuxième position.

Ces résultats peuvent constituer pour nous une bonne base de réflexion. Essayons d'explorer les facteurs qui peuvent expliquer ces divergences. 
Ils sont de plusieurs ordres. II y a d'abord des facteurs qui tiennent à la configuration du risque luimême : le politique aura intuitivement conscience de ces dimensions. Les collègues américains, dont j'ai essayé de compiler et de poursuivre le travail, appellent cela des " facteurs d'indignation ». II y a des dimensions dans le risque qui favorisent une mobilisation ou une perception plus aiguë par le public du risque et du danger.

II s'agit, par exemple, du caractère imposé ou volontaire du risque. Si l'on vous impose un risque, quelle qu'en soit l'importance en termes probabilistes, ce n'est pas la même chose que si vous décidiez délibérément de le courir vous-même. L'expérience d'une chose aussi dangereuse que la conduite automobile l'illustre parfaitement. Toutes les enquêtes de la prévention routière montrent que le risque est attribué aux autres et jamais à soi-même. On ne sera jamais indigné de courir un risque colossal qui est associé, par exemple, à la pratique du ski, parce qu'on en a décidé ainsi. En revanche, les gens qui ont la phobie des transports aériens ont l'impression de n'avoir aucune initiative ; ils pensent que la direction du véhicule leur échappe, qu'ils sont comme une allumette dans une boîte que l'on secoue. Cette dimension d'autonomie du sujet est essentielle.

À cela s'ajoute la question de savoir si le risque présente un profit quelconque pour quelqu'un, pour celui qui le subit ou pour quelqu'un d'autre. Pour les OGM, dont nous avons étudié la perception par les consommateurs français il y a un an et demi, nous avons observé en particulier que personne ne comprenait très bien quel profit l'on pouvait en tirer ; le consommateur ne voyait ainsi aucun bénéfice pour lui-même. Et d'ailleurs, dans la propagande de Monsanto et consorts, il était toujours question du tiers monde, de la faim dans le monde ou de nobles objectifs, mais jamais de bénéfices directs pour les consommateurs occidentaux. Dans la configuration de risques présentés par les OGM, le risque donc est imposé, il ne bénéficie pas au citoyen-consommateur.

J'ajouterai une troisième dimension : la dimension de maîtrise du risque, une fois qu'on a conscience de le courir. Si l'on annonçait un risque de toxicité associé à l'eau du robinet, il serait très difficile de se protéger en évitant d'utiliser cette eau, car on en a toujours besoin. La maîtrise du risque s'avère, en ce cas, impossible.

En revanche, au début de la crise de "la vache folle ", il a été annoncé que seuls les tissus neurologiques présentaient un danger, qu'il suffisait d'éviter les abats. Dans un premier temps, les personnes que nous interrogions disaient qu'il ne fallait pas manger d'abats et que, d'ailleurs, elles n'en mangeraient plus. D'autres, plus maximalistes, évitaient la viande de bœuf, en général. On avait l'impression que l'on pouvait, par des décisions assez simples, se protéger contre le risque.

La deuxième phase a été celle de la " prolifération du risque ». L'on a découvert alors qu'une foule de sous-produits du bœuf étaient utilisés dans toutes sortes d'applications industrielles alimentaires ou non alimentaires, depuis le fil chirurgical jusqu'à la gélatine dans les bonbons. La perception du public a changé alors complètement, car la maîtrise semblait impossible.

Je cite rapidement les autres dimensions que l'on analyse. Une catastrophe naturelle n'est pas assimilable à une catastrophe technique, encore que l'on ait tendance à chercher des boucs émissaires. Quand une technique inconnue est en cause, le risque est perçu comme plus aigu que lorsqu'une technique plus familière est utilisée. 
On peut établir une grille à partir de là et examiner comment se situent les différents risques qui nous ont été présentés, en grande partie d'ailleurs, par les médias.

Là encore, vous êtes au moins aussi conscients que moi du fait que la dimension médiatique est très différente, très indépendante de la dimension réelle dans I'opinion. Les médias ont deux thématiques sur ces sujets. La première figure de rhétorique médiatique est celle du scandale.

Le comble du scandale est l'affaire du Château Giscours à Bordeaux, il y a un an. On annonce qu'un grand cru classé de Bordeaux est poursuivi pour des pratiques illégales. Cette situation de scandale est typique : c'est le télescopage du noble et de l'ignoble. On est dans le comble du noble et les pratiques sont le comble de l'ignoble. Un an plus tard, il y a toujours des poursuites pour infraction à la loi sur les appellations d'origine contrôlées, mais cela concerne uniquement le second vin de Château Giscours.

Les médias ont été très sensibles à cette thématique. La tâche des news magazines est d'élargir le débat, de prendre de la distance et d'ouvrir des angles problématiques. L'hebdomadaire Marianne a titré au bout de quinze jours : "Y a-t-il encore du raisin dans le vin français ? ". C'était peut-être disproportionné par rapport aux faits avérés, à savoir l'inversion de deux cuves. On avait $160 \mathrm{hl}$ de Haut Médoc et du Margaux. Il semble que le Margaux était de moins bonne qualité que le Haut Médoc. Même si la pratique était œnologiquement défendable, elle est condamnée par la loi.

M. Gilbert Mitterrand : En Corse, l'on était parvenu à faire du vin sans raisins.

M. Claude Fischler : En termes de rhétorique médiatique, ce n'était pas dans ce cas une situation de scandale considérable. On n'a pas l'impression que, en Corse, ce soit le sommet de l'aristocratie œnologique qui se compromette dans des pratiques scandaleuses.

Je voudrais vous mettre en garde contre la seconde figure. Je me rends compte que, moi-même, j'en suis victime d'une certaine façon. Quand les médias ont bien exploité la figure rhétorique du scandale et que cela s'est un peu tassé, ils ont une seconde possibilité qu'ils ne se privent d'ailleurs pas d'exploiter en matière de sécurité alimentaire, à savoir la grande peur : celle de l'an 2000, la panique, l'irrationalité du consommateur.

Je suis sollicité en tant que sociologue. J'expliquerai brièvement les dimensions que l'on peut appeler " irrationnelles » dans notre façon de voir l'alimentation et les problèmes qui y sont liés. C'est l'une des grandes idées à retenir.

Estimer irrationnels les comportements des consommateurs, des citoyens, quand on annonce, par exemple, que 40 millions de canettes de Coca-Cola sont retirées de la vente me paraît pour le moins exagéré. J'entends à la radio que l'on retire 40 millions de canettes suite à $X$ cas de malaises; je ne vois pas ce qu'il y a d'irrationnel à m'abstenir de consommer du Coca-Cola pendant quelques jours, en attendant d'être mieux informé ou à me tourner vers la marque concurrente ! Tout cela est de bonne gestion.

Dans la première crise de "la vache folle ", on a parlé de panique, quand la consommation a baissé, puis on a dit qu'il fallait relativiser la panique, car la consommation n'avait baissé que de $6 \%$, alors qu'en Grèce, elle avait baissé de $40 \%$. 
Disraeli disait qu'il y avait trois sortes de mensonges : " les mensonges, les mensonges éhontés et la statistique ". II s'agit d'une illustration parfaite : l'illusion statistique. Si la consommation de bœuf diminue de $40 \%$ en Grèce, ce n'est pas plus important que si la consommation de bœuf en France baisse de $6 \%$. Et ce, pour une raison simple : nous disons en France que « nous gagnons notre bifteck ». Cela signifie que le bœuf est au centre de notre alimentation. De nombreux compatriotes pensent qu'ils n'ont pas mangé s'ils n'ont pas mangé de viande à un repas. C'est le cœur même de notre alimentation qui est touché. Qualitativement et quantitativement, le taux de $6 \%$ est considérable.

En revanche, une baisse de $40 \%$ de la consommation de bœuf dans un pays qui n'en mange pas ou que très peu n'est pas très importante. On peut facilement remplacer le bœuf par ce que l'on a I'habitude de consommer, du mouton ou autre chose. Cette lecture des statistiques est complètement trompeuse.

Il y a aussi un rapport coût-bénéfice rationnel chez le consommateur : j'évite de consommer quelque chose qui m'est présenté comme dangereux, même si la probabilité du risque est infinitésimale. Je fais quand même un calcul implicite extrêmement rationnel. Je m'abstiens de consommer de l'huile de colza, dans laquelle on m'a dit qu'il y avait de l'acide érucique qui pouvait provoquer des troubles cardiologiques. Qu'est-ce que cela me coûte de renoncer à l'huile de colza, puisque cela ne fait pas partie de mon répertoire alimentaire ? Je ne me prive pas et, en échange, je me protège contre certains problèmes.

Nous avons affaire là à des calculs aussi bien irrationnels que rationnels qui ne sont pas aussi simples que les médias veulent nous le dire. Disons qu'il s'agit d'une autre rationnalité.

Pour finir sur ce point, je voudrais évoquer deux idées. Les principaux changements dans les pratiques alimentaires des Français tiennent au fait que des filières se sont installées. Une transformation croissante des aliments s'est produite depuis quelques décennies et l'apparition de la grande distribution remonte au début des années 70 . L'industrialisation de l'alimentation est postérieure à la Seconde Guerre mondiale. Elle entraîne des conséquences considérables sur la perception par nos compatriotes de l'alimentation.

Pour caricaturer, il y a " jadis et aujourd'hui ». Jadis, on pouvait avoir une connaissance presque intime des produits alimentaires que I'on consommait parce qu'une très grande partie de ces produits poussait au pied de nos habitations. Les viandes venaient elles aussi d'un écosystème presque immédiat. De plus en plus, nous sommes dans un système où une distance croissante existe entre le stade de la production et celui de la consommation.

Je dis volontiers que les produits alimentaires transformés par l'industrie sont devenus des " objets comestibles non identifiés ". Ils sont " packagés ", conditionnés, " marketés ", " publicisés » et nous ignorons tout de leur origine, des process subis, des transformations. Cela ressort des entretiens des enquêtes et des réunions de groupes. D'où un changement radical dans notre rapport à ces aliments et un besoin frénétique d'avoir plus d'informations.

Mais il s'agit d'un besoin jamais satisfait, car il est probablement difficile à satisfaire. "Nous ne savons pas ce nous mangeons " est la phrase que j'entends le plus souvent dans les enquêtes. Je vous demande de réfléchir sur le sens de cette phrase qu'il faut prendre au sens littéral. 
Pourquoi est-il si important de savoir ce que l'on mange ? J'aborde le caractère que j'avais annoncé « irrationnel » de l'alimentation. Nous savons de manière expérimentale - c'est un fait scientifique avéré - qu'il y a une part de pensée magique dans notre rapport au monde et à l'alimentation en particulier.

La pensée magique consiste en deux choses : en premier lieu, la pensée de la contagion. Si je touche quelque chose, il passera en moi-même quelque chose de pollué, de souillé. II faudra des rites pour s'en débarrasser. Il y a aussi l'idée que l'image égale l'objet. Je prends une photo et je la soumets à des sorts.

Vous pouvez vous demander ce que cela a à voir avec un pays développé au xxi ${ }^{\mathrm{e}}$ siècle. La réponse est que nous avons des preuves expérimentales que tout le monde fonctionne de cette manière. Si je vous demande de déchirer la photo de vos enfants, vous ne pourrez pas le faire de gaieté de cœur, même si vous possédez les négatifs ou un double. En revanche, les politiques savent mieux que personne que, dans les manifestations populaires, on a l'habitude de pendre ou de brûler l'image de certaines personnes en effigie, et que l'on n'a pas beaucoup de mal à mettre des moustaches sur la représentation de quelqu'un que l'on n'aime pas. Ce sont là des manifestations de pensée magique.

Dans le domaine de l'alimentation, nous avons des preuves expérimentales que l'ensemble de la population - ce n'est nullement une question d'arriération ou d'irrationalité - possède cette croyance très profondément ancrée que, si nous mangeons quelque chose, cela va nous transformer de l'intérieur et que nous allons devenir analogues à ce que nous mangeons.

La publicité fonctionne beaucoup en matière alimentaire sur ce modèle-là. L'eau d'Évian vous donne, par exemple, ce que la montagne lui a donné. La barre "Lion " de Nestlé fait apparaître un lion à l'image ; quand j'arrive au bureau et que je suis très en forme, on me demande si j'ai « mangé du lion ». Le langage transmet cette histoire.

Pardonnez-moi de parler de choses apparemment aussi éloignées des questions graves, politiques et économiques qui vous préoccupent, mais c'est fondamental dans la perception de nos contemporains. Si on me dit toujours que l'on ne sait pas ce que l'on mange, c'est clairement parce que, si « je suis ce que je mange » et que « je ne sais plus ce que je mange », je ne sais plus qui je suis.

Une inquiétude très profonde s'associe à tout cela, j'en vois la preuve constante dans toutes les enquêtes. C'est un phénomène sans doute universel qui, chez nous - pays à forte tradition culinaire et gastronomique -, prend une dimension tout à fait particulière.

D'autres travaux que je serais ravi de vous communiquer montrent que nous n'appréhendons pas l'alimentaire de la même façon que nos voisins. Nous sommes plutôt moins angoissés que les autres. Nous avons plutôt moins de préoccupations dans nos choix alimentaires, directement dictées par les considérations de santé. Nous considérons l'alimentation en termes de plaisir, de convivialité, de sociabilité, alors que nos voisins du Nord de l'Europe, Anglo-Saxons et Américains exercent leurs choix alimentaires avec des préoccupations essentiellement sanitaires que l'on ne voit pas apparaître ici. 
Cette particularité doit impérativement être prise en compte. II ne faudrait pas jeter le bébé avec l'eau du bain et, par souci de sécurité ou de se rassurer, entrer excessivement dans des logiques qui nous mèneraient à certaines pratiques ou à certaines attitudes couramment observées en Grande-Bretagne ou aux États-Unis où se manifeste une sorte d'obsession quant aux sources de contamination bactérienne et à la nutrition.

Les préoccupations varient selon les pays. Les Américains sont beaucoup plus soucieux que nous des sources de contamination bactérienne. Leur vision du corps est celle d'une sorte de citadelle assiégée par des ennemis extérieurs et, pour eux, il faut protéger toutes les ouvertures contre les assauts bactériens. Pour des raisons historiques, nous avons une vision qui est plus I'héritage de la médecine hippocratique avec l'équilibre interne, les tempéraments, les humeurs. Il est d'ailleurs fascinant de voir que les effets de divergences historiques de trois ou quatre cents ans se font sentir aujourd'hui dans notre rapport à la santé et à l'alimentation.

Nous tenons ici à la dimension plaisir et sociabilité de l'alimentation. Ceci n'est d'ailleurs pas remis profondément en cause par les événements récents. Les Français sont généralement attachés à la notion de qualité qui est pour eux quasiment synonyme de sécurité. Après " la vache folle ", ils se sont tournés vers les signes de qualité, contrairement à beaucoup de leurs voisins européens.

M. le président : Nous avons été très intéressés par votre exposé. Des questions complémentaires permettront encore d'éclairer les sujets qui nous préoccupent.

M. Gilbert Mitterrand : J'essayais de résumer tout ce qui a été dit ce matin par vous-même et par d'autres intervenants. Je ne parle pas de ce qui est resté stable dans le pays avec son mode d'alimentation venant du potager, mais des mutations qui ont touché d'autres pans de la société française.

Il est certain que, lorsqu'on mange, cela ressort plus d'une nécessité dont le mode est imposé. On est pressé, on se déplace beaucoup, on va plus facilement au restaurant, le convivial familial a changé. C'est notre mode de vie qui a engendré toutes ces évolutions. Par conséquent, on devrait faire davantage confiance à ce que l'on mange, puisqu'on le contrôle moins. Or ce n'est pas ce sentiment-là qui ressort. À vous entendre, on subit la qualité de la nourriture, on ne la contrôle pas.

Deuxièmement, on a l'idée que cette restauration qui s'est adaptée à notre nouveau mode de vie recherche plus le profit économique que le bien-être de la personne alimentée. II y a cette double contrainte, d'un côté, de manger ce qui se présente et, de l'autre côté, de bien comprendre que tout cela se passe dans un système économique où le souci du profit l'emporte sur celui du bien-être des uns et des autres.

Finalement, ces deux rencontres ne font-elles pas que l'on subit et que l'on accepte ? Pour ne plus subir et accepter en même temps, sociologiquement, quels sont les signes forts ? D'où peuvent-ils venir ? En quoi ont-ils des chances d'atteindre leur cible ? À part les médias qui, comme vous l'avez dit tout à l'heure, ne procèdent qu'au travers des scandales.

Je suis parlementaire de la région libournaise et je sais que l'on peut frauder de façon extraordinaire, mais que ce ne sont pas les grandes $A O C$ qui font cela. 
Nous aurons des propositions, des suggestions à faire. Dans le contexte économique et sociologique que vous connaissez, comment ces suggestions ont-elles une chance d'être entendues ? Par une loi ? Mais il y en a tellement. À moins qu'elle ne porte en elle un message plus fort que son contenu.

M. Claude Fischler : Votre exemple sur la restauration est tout à fait juste. Nous sommes conscients qu'il ne s'agit pas que de la restauration. La restauration hors domicile est moins importante en France que dans les pays voisins.

Le CREDOC nous livre ce chiffre étonnant : $60 \%$ des Français mangent encore chez eux à midi. En revanche, les produits alimentaires transformés par l'industrie concernent tout le monde. Ils sont disponibles dans la grande distribution. C'est à ce niveau-là que nous avons une conscience partagée.

D'un côté, nous utilisons les produits transformés par l'industrie, dans lesquels nous trouvons des bénéfices de prix, de commodité d'emploi et même de sécurité pour certains d'entre nous.

Mais, dans le même temps, si nous pensons avoir gagné une chose, nous en avons perdu une autre. Ce quelque chose, c'est le goût, la qualité jubilatoire de l'alimentation quotidienne, autrement dit une dimension de sociabilité. On n'a plus le temps, on ne prend plus le temps d'être ensemble.

Le sentiment que l'on ne peut rien faire contre cela s'impose dans les esprits. La phrase qui revient le plus souvent est : " On n'arrête pas le progrès. » II est intéressant de voir à quel point la signification du mot " progrès " a pu évoluer. Avant, il s'agissait surtout d'émerveillement ; maintenant, l'on éprouve plutôt de la résignation, en tout cas pour l'alimentation. Je ne dis pas que j'obtiendrais les mêmes résultats à propos des nouvelles technologies de l'information.

L'impression dominante est celle d'une conscience partagée, contradictoire, un peu positive, un peu négative, avec du tiraillement. Dans les périodes calmes, on se réconcilie plus ou moins avec ces produits alimentaires transformés par l'industrie. Au moindre déclic, histoire de colorant ou de " vache folle ", c'est la crise, l'explosion ; l'inquiétude réelle se redéclenche, en attendant que les choses se calment à nouveau provisoirement.

Si l'on comprend bien ce phénomène et que, par ailleurs, on a en tête tout ce que j'ai essayé de dire sur la perception, sur le caractère magique de notre façon de penser les choses et sur cette identité liée profondément à l'alimentation, les positions à prendre sont aisées à définir.

Il me semble que tout le problème consiste à ré-identifier les aliments dans la tête du consommateur. Il faut retrouver comment les produits alimentaires que nous consommons sont arrivés là, ce qu'ils ont subi, de façon à pouvoir se rassurer soi-même sur les effets que leur consommation aura sur notre identité, sur celle de nos proches.

Que faire à ce sujet ? Sur le plan législatif, je vous laisserai tirer les conclusions de ce que je dis, car je ne suis pas habitué à raisonner en termes réglementaires ou législatifs. II y a des choses qui existent déjà, notamment un attirail extraordinaire d'artefacts destinés à aider le consommateur à identifier ses aliments, il y a la marque qui est une façon pour lui de mettre un sceau sur le produit, associé à un nom, qui lui-même a une image. On sait ce que l'on achète ou on croit le savoir, quand on achète une marque. 
On sait expérimentalement que la puissance de la marque est extraordinaire. Si je donne à déguster en aveugle du Coca-Cola ou du Pepsi-Cola, 51 \% des gens préfèrent le Pepsi-Cola. Si je leur dis la marque, la préférence s'inverse. Chez Pepsi, on dit toujours que, pour faire fortune très rapidement, il faudrait vendre du Pepsi dans une bouteille de Coca et, pour se ruiner, vendre du Coca dans une bouteille de Pepsi. La marque est un instrument d'une puissance extraordinaire comme les placebos en médecine.

Deuxièmement, il y a les labels, les signes de qualité. Les Français leur font confiance. Pourquoi ? Dans la perception de l'aliment par les Français, en opposition à leurs voisins, il y a une dimension holistique globale qui peut s'exprimer ainsi : "Si c'est bon, cela ne peut pas vraiment faire de mal. " II ne s'agit pas en ce cas de toxicité ; quand, après 50 ans, on sait que l'on a du cholestérol, on dit le contraire : « tout ce qui est bon me fait du mal ».

Quand on interroge les Français pour savoir comment ils choisissent leur viande, ils répondent que la viande doit être belle, tendre et goûteuse et que, pour avoir de la bonne viande, il faut aussi choisir un bon boucher et établir des liens très intimes avec lui. Les Français se tournent vers les signes de qualité, y compris quand ils ont des problèmes de sécurité ; cela a d'ailleurs été caractéristique pour « la vache folle $»$.

Les Français sont persuadés que qualité, sécurité, goût, plaisir relèvent du même combat ; ce n'est pas du tout ce que pensent les Scandinaves ou les Américains.

Nous abordons là les aspects législatifs ou réglementaires. Aux États-Unis, il y a un an, la FDA (Food and Drug Administration) nous a présenté l'arsenal de son étiquetage. On se rend compte que, derrière cela, il y a une utopie politique, différente de la nôtre, qu'il faut analyser. Aux États-Unis, l'étiquetage de la FDA impose de faire figurer tous les ingrédients dans des conditions extrêmement strictes : l'ordre, le type...

Ensuite, il y a une autre recommandation qui est la RDA (Recommanded daily allowance) : quel pourcentage des apports conseillés représente chacun des ingrédients en question dans votre alimentation ?

En troisième lieu, la communication du USDA (US Department of Agriculture), le ministère de l'Agriculture américain, porte sur la pyramide nutritionnelle. II s'agit des directives et des conseils nutritionnels à l'usage du peuple américain. En bas de la pyramide, il y a les choses qu'il faut consommer plusieurs fois par jour et, en haut de la pyramide, ce qu'il faut consommer rarement. En bas, il y a les féculents, les légumes et les fruits frais ; en haut, la viande, le sucre et les matières grasses.

Représentons-nous le consommateur américain. II va aller dans son supermarché prendre un produit ; il va regarder les ingrédients, les pourcentages des apports nutritionnels conseillés. II va rapporter tout cela aux nutritional guidelines. Il possède sans doute un ordinateur qui compilera tous ces éléments.

Le rôle de l'État, face à des citoyens qui sont des individus libres et égaux en droits, est de fournir une information vraie et rationnelle, fondée en science, car seule la science peut fournir la vérité et la raison. Donc, on va fournir au consommateur toute cette information et lui dire de faire ses calculs et de se composer une alimentation rationnelle et équilibrée. 
Cela repose sur des prémisses qui sont fausses : manger résulterait d'une somme de décisions individuelles quotidiennes qui sont prises rationnellement. Vous savez comme moi que ce n'est pas ainsi que cela fonctionne. Si l'on mange ce que l'on mange, ce n'est pas parce qu'on a fait peser, calculer, c'est parce qu'on appartient à une culture qui fait qu'on mange à telle heure, que le repas est fait de tels ingrédients, que l'on mange dans tel endroit et non dans un autre. C'est ainsi que l'on a appris, que l'on fait.

Comme dans le langage, se nourrir a une grammaire. Quand je parle ma langue, je ne pense pas à chaque instant où sont le sujet, le verbe et le complément. Je le fais. Quand je mange, c'est identique : j'applique des règles sociales et culturelles dont je n'ai conscience que lorsqu'elles sont transgressées. Les Américains ont une attitude de dénégation complète par rapport à cela. Les Français ont au contraire cette culture de la « bouffe » en eux et cela gouverne beaucoup leurs attitudes.

Par conséquent, face aux problèmes que vous soulevez, je pense que la meilleure stratégie consiste à ré-identifier les produits autant que possible : les signes de qualité, les normes, les marques, l'étiquetage informatif. Quand on demande aux gens s'ils souhaitent avoir des informations, ils répondent qu'ils en veulent toujours mais qu'ils ne les lisent jamais, à l'exception des femmes qui sont toujours plus angoissées que les hommes. II y a une différence radicale dans la perception du risque entre les hommes et les femmes. Les femmes ont une perception aiguë des risques, les hommes étant beaucoup plus tranquilles, en matière d'alimentation.

\section{Gilbert Mitterrand : Ils font peut-être confiance aux femmes !}

M. Claude Fischler : L'étiquetage informatif n'est donc pas tellement " conforme » du point de vue français. Même les labels comme le " bio " rencontrent une adhésion relative quand s'y associent la qualité et l'appétence. Le " bio " de jadis était peu appétissant. C'était bon pour des gens dyspeptiques. Aujourd'hui, sa présentation s'est améliorée et, avec les nouvelles préoccupations de qualité, il se vend beaucoup mieux.

Je pense qu'il faut aller au fond des choses. La seule manière d'aider à régler ce problème de crédibilité des aliments est d'assumer les changements dans le mode de production agro-alimentaire. II faut incontestablement que les citoyens et les consommateurs aient de plus en plus conscience du fait que le mode de production que nous avons connu pendant quelques décennies est remis en cause par les opérateurs, par les politiques et même par tout le monde, qu'un vaste processus de changement est en cause, qu'il y a un débat et qu'on va peut-être revenir sur certaines pratiques. C'est la seule solution de fond que l'on puisse envisager, si elle est praticable.

M. Gilbert Mitterrand : Même si, par des obligations de quelque ordre qu'elles soient, on apportait des réponses en matière de garantie, de sécurité, de traçabilité, d'étiquetage, de label, d'origine contrôlée dans un circuit de grande distribution, diriez-vous que, sociologiquement, les Français restent des sceptiques ? Feraient-ils vraiment confiance? 
M. Claude Fischler : En France, les mesures auxquelles vous faites allusion pourraient sans doute améliorer la perception des usagers, mais elles seraient incapables de régler le problème si, par ailleurs, les consommateurs n'avaient pas la conviction qu'il y a des changements réels et profonds dans le mode de production agro-alimentaire.

M. le président : Vous avez parlé du comportement des Anglo-Saxons et de leur souci d'éviter les contagions bactériennes. Cela n'est-il pas contradictoire, s'agissant du « bœuf aux hormones » ou de I'ESB, avec le fait qu'ils semblent en retrait par rapport à nos préoccupations sur ces questions ?

M. Claude Fischler : On me pose souvent la question. La réponse est simple. II n'est pas vrai qu'ils ne se préoccupent pas du contenu de leur alimentation. Pour les OGM, les enquêtes dont il a été fait état ont été commanditées et exécutées par Monsanto. J'ai assisté à une présentation de résultats par Monsanto à la FDA, où l'on nous expliquait que les consommateurs américains n'étaient pas inquiets des OGM. II y a des questions induites ou inductives et des résultats peuvent être analysés et présentés d'une manière ou d'une autre.

Quand nous avons fait une enquête sur la perception des OGM par les Français, si Monsanto avait communiqué les résultats que nous avons obtenus, il aurait probablement dit la même chose des Français. Que trouvions-nous ? Nous trouvions que les Français ne savaient pas ce qu'étaient les OGM. Donc, ils ne s'en préoccupaient guère. Ils ne savaient pas ce qu'étaient les OGM car ils ne faisaient pas la différence entre ce qui est manipulé génétiquement et ce qui est produit par les méthodes classiques de sélection. Pour eux, c'était plus ou moins la même chose que les aubergines blanches ou les courgettes courbées. On observait une certaine incompréhension du phénomène.

Axel Kahn a démissionné de sa présidence de la commission du génie génétique, entre autres suite à une réunion en présence de la presse où il s'était agacé du fait qu'ayant présenté aux médias l'état des connaissances en matière d'OGM les journalistes lui disaient : "Que voulez-vous que l'on dise dans les journaux à ce sujet ? I| n'y a rien à dire ! »

Tant qu'une crise n'a pas éclaté, « il n'y a rien à dire ». Si l'on n'est pas dans la figure du scandale ou de la grande peur, il n'y a pas grand-chose à dire pour les médias. Pour le public, on ne sait pas de quoi il s'agit. Si on renvoie à des processus sur lesquels il existe déjà une perception établie - comme le progrès que l'on n'arrête pas - on peut très bien dire, si on présente les résultats de façon tendancieuse, qu'il n'y a pas d'inquiétude particulière dans l'opinion. Tant que ce n'est pas déclaré, actuel, présent, ce n'est pas vraiment un problème.

Finalement, les Américains se sont réveillés ces derniers temps. II y a eu des renversements considérables en deux mois. Les filières sans OGM surgissent partout. Les organisations de consommateurs aux États-Unis sont beaucoup plus sensibles qu'on ne l'a dit en matière de viande aux hormones.

Il y a deux restrictions : la première est qu'ils sont habitués depuis plus longtemps que nous à une alimentation transformée par l'industrie. II y a chez eux une familiarité plus grande avec les produits transformés. On se rend compte que certains processus - que j'ai évoqués pour la France - sont très importants. II s'agit de l'attachement aux marques. Coca-Cola par exemple a un contenu extraordinairement affectif. 
Quand Coca-Cola a voulu changer la composition de son produit, il y a eu une véritable révolte des consommateurs. Même les Américains sont sensibles à une forme de continuité ou de tradition, y compris dans le contexte industriel.

La seconde restriction concerne ce que je vous disais à propos des germes, des microbes et des contaminations bactériennes. Certains éléments culturels spécifiques font que les Américains, comme les Anglais, sont beaucoup plus inquiets que nous des contaminations bactériennes. Cette particularité culturelle ne concerne pas seulement la population en général, mais les scientifiques eux-mêmes. On le constate très bien lors des débats sur les fromages au lait cru à l'échelon européen.

Les experts anglais n'imaginent pas qu'on puisse vendre dans la grande distribution des fromages au lait cru fermenté et ils n'arrivent pas davantage à imaginer qu'on puisse vendre des plats préparés sous vide à relativement basse température. Ce qui est vendu en France au rayon frais sous atmosphère contrôlée et cuisson sous vide à basse température paraît très risqué aux Anglo-Saxons et aux Scandinaves. Les différences culturelles existent.

M. le président : Il y aurait donc une différence de perception entre Latins et gens d'Europe du Nord.

M. Claude Fischler : C'est clair. Je peux vous montrer les résultats d'une enquête qui le confirment. À titre d'exemple, nous avons fait récemment des réunions de groupes de consommateurs sur la viande en France, en Italie, en Grande-Bretagne et en Finlande.

Entre les groupes français et italien, il y avait des points communs considérables : l'attachement à la qualité, la stratégie de reconnaissance de la qualité : il faut un bon boucher et être très copain avec lui. Leur principale préoccupation était la qualité. Pour les Anglais, le groupe a essentiellement discuté de deux choses : faut-il ou non être végétarien ? Deuxièmement, si on est végétarien, comment fait-on pour se nourrir quand on va sur le continent?

Quant aux Finlandais, l'animatrice nous a dit qu'elle avait passé un moment épouvantable. Pendant trois heures, elle a essayé de les faire parler de la viande comme aliment. Tout ce qu'elle a réussi à obtenir s'est résumé à une discussion sur la question de savoir s'il était bien de tuer les animaux ou non et si cela faisait une différence de les tuer à la chasse ou à l'abattoir. II s'agissait de questions uniquement éthiques.

Cela reflète des points de vue culturels radicalement différents. En Angleterre, il y a entre 8 et $12 \%$ de végétariens de diverses obédiences. Chez nous, ils sont tellement rares que l'on ne parvient pas à les compter. Tout cela est ancré dans des traditions, elles-mêmes pour beaucoup liées à la religion. Le végétarisme anglais commence au xviii ${ }^{\mathrm{e}}$ siècle avec une fraction progressiste libérale du clergé anglican. À partir du moment où vous avez 8 à $12 \%$ de végétariens sur un marché, cela devient intéressant pour l'industrie. On entre dans un cycle d'entretien ou d'accentuation du phénomène. Pour l'instant, on n'a rien de tout cela en France et je ne vois pas que cela s'annonce.

M. le président : Vous avez fait référence à certaines enquêtes. Pourrait-on disposer de ces enquêtes, M. le directeur? 
M. Claude Fischler : Je serais ravi de vous faire passer les tirés à part. Il s'agit d'une enquête comparative sur quatre échantillons : des Français, des Américains, des Japonais et des Belges néerlandophones. Elle n'a été publiée qu'en anglais dans une revue scientifique [1]..

\section{REFERENCES}

1. RAZIN P, FISCHLER C, IMADA S, et al. (1999). Attitudes to food and the role of food in life in the USA, Japan, Flemish Belgium and France : Possible implications for the Diet-Health Debate. Apetite, 33 : $163-$ 80.

* Audition de M. Claude Fischler (extrait du procès-verbal de la séance du mardi 2 novembre 1999), devant la commission d'enquête parlementaire sur la transparence et la sécurité sanitaire de la filière alimentaire en France. Présidence de M. Félix Leyzour, rapporteur M. Daniel Chevallier. 